\title{
Relativistic Mean Field Approach and the Pseudo-Spin Symmetry
}

\author{
G. A. Lalazissis ${ }^{1}$, Y. K. Gambhir ${ }^{1,2}$, J. P. Maharana ${ }^{2}$, C. S. Warke ${ }^{2}$, and P. Ring ${ }^{1}$ \\ ${ }^{1}$ Physik-Department der Technischen Universität München, D-85747 Garching, Germany \\ 2 Department of Physics, Indian Institute of Technology Bombay \\ Powai, Mumbai 400026, India
}

October 20, 2018

\begin{abstract}
Based on the Relativistic Mean Field (RMF) approach the existence of the broken pseudo-spin symmetry is investigated. Both spherical RMF and constrained deformed RMF calculations are carried out employing realistic Lagrangian parameters for spherical and for deformed sample nuclei. The quasi - degenerate pseudo-spin doublets are confirmed to exist near the fermi surface for both spherical and deformed nuclei.
\end{abstract}

PACS numbers : 21..60Cs, 21.10.-k, 24.10.Jv

Pseudospin symmetry has been discovered in nuclear physics nearly 30 years ago [1 3 ]. The recent claim [4 that pseudo-spin symmetry may arise due to near equality in magnitude of attractive scalar and repulsive vector fields in relativistic mean theory, has revived the activity related to the understanding of the origin of this symmetry in real nuclei. The concept of pseudo-spin symmetry [1,2] is based on the experimental observation of the existence of quasi-degenerate doublets of normal parity orbitals $\left(n, \ell, j=\ell+\frac{1}{2}\right)$ and $(n-1$, $\left.\ell+2, j=\ell+\frac{3}{2}\right)$ such as $\left(4 s_{1 / 2}, 3 d_{3 / 2}\right),\left(3 d_{5 / 2}, 2 g_{7 / 2}\right)$ etc., in the same major shell. Since for spherical systems the quantum numbers $j^{\pi}$ are conserved, the pseudo-spin angular momenta $(\tilde{\ell}, \tilde{s}=1 / 2)$ satisfy $\tilde{j}=j=\tilde{l} \pm \frac{1}{2}$.

In order to interpret this near degenerate pair of $j=\ell+1 / 2$ and $j=\ell+3 / 2$ states as pseudo-spin doublets corresponding to $\tilde{m}_{s}= \pm 1 / 2, \tilde{\ell}$ has to be $\ell+1$. It then follows for the major oscillator quantum number: $\tilde{N}=N-1$, for the radial quantum number $\tilde{n}=(\tilde{N}-\tilde{\ell})-1$ and for the parity $\tilde{\pi}=-\pi$. For zero pseudo-spin orbit splitting, the pseudo- 
spin multiplet will be degenerate. Thus the pair of orbitals $\left(4 s_{1 / 2}, 3 d_{3 / 2}\right)$ and $\left(3 d_{5 / 2}, 2 g_{7 / 2}\right)$ can be viewed as the $\left(2 \tilde{p}_{1 / 2}, 2 \tilde{p}_{3 / 2}\right)$ and $\left(1 \tilde{f}_{5 / 2}, 1 \tilde{f}_{7 / 2}\right)$ pseudo-spin doublets. The symmetry can also be investigated in deformed nuclei. In the asymptotic Nilsson scheme one finds the pseudo-spin quantum numbers $\left(\tilde{N}=N-1, \tilde{n}_{3}=n_{3}, \tilde{\Lambda}=\Lambda+1\right.$ and $\left.\tilde{\Omega}=\Omega\right)$. Therefore, the Nilsson orbitals $\left[N, n_{3}, \Lambda, \Omega=\Lambda+1 / 2\right]$ and $\left[N, n_{3}, \Lambda+2, \Omega=\Lambda+3 / 2\right]$ can be viewed as the pseudo spin-orbit doublets $\left[\tilde{N}, \tilde{n}_{3}, \tilde{\Lambda}, \tilde{\Omega}=\tilde{\Lambda} \pm 1 / 2\right]$ [5].

Apart from the rather formal relabeling of quantum numbers various proposals for an explicit transformation from the normal scheme to the pseudo-spin scheme have been made in the last twenty years and several nuclear properties have been investigated in this scheme [6 9]. However, the origin of pseudo-spin symmetry remained unknown until the recent observation of Ginocchio [4, 10] where for the first time the origin of this symmetry is claimed to be revealed as due to the near equality in magnitude of the attractive scalar and repulsive vector fields in relativistic theories. Here in this letter we follow this idea and investigate to what extent the pseudo-spin symmetry is broken for realistic cases. For this purpose we concentrate as well on spherical as on deformed nuclei and we use the framework of relativistic mean field (RMF) theory [11]. It has been shown that this phenomenological approach is very successful in describing the ground state nuclear properties of spherical, deformed and also for nuclei far away from the beta stability line (see for example [12 14].

The RMF starts with a Lagrangian density describing the nucleons as Dirac spinors $\psi$, of mass $m$, interacting via the meson $\left(\sigma_{-}, \omega-\right.$, and $\left.\rho-\right)$ and the electromagnetic fields. The standard Lagrangian density used in the RMF theory is written as [13]:

$$
\begin{aligned}
\mathcal{L}= & \bar{\psi}\left(\gamma\left(i \partial-g_{\omega} \omega-g_{\rho} \vec{\rho} \vec{\tau}-e A\right)-m-g_{\sigma} \sigma\right) \psi \\
& +\frac{1}{2}(\partial \sigma)^{2}-U(\sigma)-\frac{1}{4} \Omega_{\mu \nu} \Omega^{\mu \nu}+\frac{1}{2} m_{\omega}^{2} \omega^{2} \\
& -\frac{1}{4} \overrightarrow{\mathrm{R}}_{\mu \nu} \overrightarrow{\mathrm{R}}^{\mu \nu}+\frac{1}{2} m_{\rho}^{2} \vec{\rho}^{2}-\frac{1}{4} \mathrm{~F}_{\mu \nu} \mathrm{F}^{\mu \nu}
\end{aligned}
$$

It includes a nonlinear self-interaction $U(\sigma)$ of the $\sigma$-field: 


$$
U(\sigma)=\frac{1}{2} m_{\sigma}^{2} \sigma^{2}+\frac{1}{3} g_{2} \sigma^{3}+\frac{1}{4} g_{3} \sigma^{4}
$$

which takes into account in a phenomenological way the density dependence of the parameters of the model. $m_{\sigma}\left(g_{\sigma}\right), m_{\omega}\left(g_{\omega}\right), m_{\rho}\left(g_{\rho}\right)$ are the respective meson masses (coupling constants) and $g_{2}$ and $g_{3}$ are the coupling strengths of the nonlinear sigma field $U(\sigma)$.

It is straightforward to write the coupled baryon spinor and the mesons mean field equations. Starting from the Dirac equation for the single nucleon radial wave function with the spherical attractive scalar $\left(S=-g_{\sigma} \sigma\right)$ and the repulsive vector $\left(V=g_{\omega} \omega\right)$ potentials and following the standard procedure, by eliminating the small components $\left(g_{i}\right)$, the large components $\left(f_{i}\right)$ obey the following second order differential equation:

$$
\begin{aligned}
& \left\{-\nabla^{2}-\frac{S^{\prime}+V^{\prime}}{2 m-E-(S+V)}\left(\frac{\partial}{\partial r}+\frac{\kappa_{i}+1}{r}\right)\right\} f_{i} \\
= & -(2 m-E-(S+V))(E-(S-V)) f_{i} .
\end{aligned}
$$

Here the eigenvalues denoted by $\kappa_{i}$, of the operator $-\beta(\boldsymbol{\Sigma} \cdot \mathbf{L}+1)$ are given by

$$
\kappa_{i}=\mp\left(j_{i}+\frac{1}{2}\right) \quad \text { for } \quad j_{i}=\ell_{i} \pm \frac{1}{2}
$$

and $S^{\prime}\left(V^{\prime}\right)$ are the derivatives of the potentials $S(V)$ with respect to $r$. The binding energy $E \geq 0$ is measured with respect to the nucleon mass $M$ in natural units $\hbar=c=1$.

On the other hand eliminating the large component $f_{i}$ we have for the small component $g_{i}$ the following second order differential equation:

$$
\begin{aligned}
& \left\{-\nabla^{2}-\frac{S^{\prime}-V^{\prime}}{E-(S-V)}\left(\frac{\partial}{\partial r}-\frac{\kappa_{i}-1}{r}\right)\right\} g_{i} \\
= & (2 m-E-(S+V))(E-(S-V)) g_{i} .
\end{aligned}
$$

For the case of equal strengths, $S=V$, the Eq. (5) reduces to:

$$
-\nabla^{2} g_{i}+E(S+V) g_{i}=E(2 m-E) g_{i}
$$

Clearly Eq. (6) has an energy dependent potential $(E(V+S))$ and has the eigenvalue $E(2 m-E)$. After scaling the radial variable $r=x /(\sqrt{E})$, the potential has a complicated 
$(\sqrt{E})$ dependence i.e., $S(x / \sqrt{E})+V(x / \sqrt{E})$. In such a situation this equation (6) is no longer a normal Schrödinger eigenvalue equation. Further, it is obvious that in this equation all solutions with "bound" states in the Fermi sea with $E \geq 0$ are shifted to one degenerate eigenvalue with $E=0$, which, in fact, is not bound. The corresponding wave functions are not normalizable. This indeed is an unphysical situation. This equation is the same as the equation (3) of Ref. [4] in the scaled variable $x$ when written in terms of the partial waves and using the relation $\ell(\ell+1)=\kappa(\kappa-1)$. Here $\ell$, the angular momentum of the lower component $g_{i}$ is identified with the pseudo-spin angular momentum $(\tilde{\ell})$. This is the pseudo-spin symmetry limit of Ref. [4], where the doublets $j=\tilde{\ell} \pm 1 / 2$ with the same $\tilde{\ell}$ are degenerate. However, in this limit only the Dirac sea states exist and no Dirac valence bound states and therefore contradicts reality. According to these considerations in all realistic situations the pseudo-spin symmetry must be broken. Therefore the question arises, to which extent it is broken in real nuclei. So far only the spherical case has been investigated for square well potentials [4] and for spherical solutions of the RMF equations [15,16].

In the present letter we investigate the broken pseudo-spin symmetry both for the spherical and deformed nuclei within the relativistic mean field approach. For our study, we choose ${ }^{208} \mathrm{~Pb}$ as a representative of spherical nuclei and ${ }^{154} \mathrm{Dy}$ as a representative of deformed nuclei. We use in our calculations the Lagrangian parameter set NL3 [17 which successfully reproduces the ground state properties of nuclei, spread over the entire periodic table. The other parameter sets like NL1 and NLSH (see [14]) are expected to give almost identical results for these nuclei.

First, spherical RMF calculations in the coordinate space are carried out for ${ }^{208} \mathrm{~Pb}$. The calculated binding energy and the charge radius agree remarkably well with the experiment. The calculated single particle energies for the bound orbitals near the fermi surface are shown in Fig. ([a) for neutrons and protons. It is clear from the figure that the pairs of bound neutron valence orbitals $\left(2 g_{7 / 2}, 3 d_{5 / 2}\right)$ and $\left(1 i_{11 / 2}, 2 g_{9 / 2}\right)$ which correspond to pseudo-spin 
doublets $\left(2 \tilde{f}_{7 / 2}, 2 \tilde{f}_{5 / 2}\right)$ and $\left(1 \tilde{h}_{11 / 2}, 1 \tilde{h}_{9 / 2}\right)$ respectively, are quasi-degenerate indicating only a small breaking of pseudo-spin symmetry. The same is more or less true for the pairs of neutron hole $\left(\left(2 f_{5 / 2}, 3 p_{3 / 2}\right),\left(1 h_{9 / 2}, 2 f_{7 / 2}\right)\right)$, proton valence (particle) $\left.\left(1 h_{9 / 2}, 2 f_{7 / 2}\right)\right)$, and proton hole $\left(\left(2 d_{3 / 2}, 3 s_{1 / 2}\right),\left(1 g_{7 / 2}, 2 d_{5 / 2}\right)\right)$, orbitals forming the pseudo-spin doublets. But here the energy separation between the partners of the respective doublets is relatively larger. The larger is the binding energy the larger is the separation. This indicates that the concept of the pseudo-spin symmetry becomes better and better for the orbitals as their energies approach closer and closer to the continuum. This is consistent with the results found in Ref. 四 for the square well potentials. In addition, the energy separation becomes larger, if the pseudo-orbital angular momentum $(\tilde{\ell})$ increases. The dependence of the energy splitting of the pseudo-spin partners on the energy $\mathrm{E}$ and on the pseudo-orbital angular momentum $\tilde{\ell}$ can easily be understood from Eq.(5). For a given pseudo-orbital angular momentum $\tilde{\ell}$ the term in Eq.(5) which splits the pseudo-spin partners is:

$$
\frac{S^{\prime}-V^{\prime}}{(S-V)-E} \frac{\kappa_{i}}{r}
$$

It has the energy dependence $(E-(S-V))$ in the denominator. Now $(S-V)$ is about $50 \mathrm{MeV}$. Bound states in the Fermi sea have a binding energy $E<50 \mathrm{MeV}$. For increasing binding energy $E$, i.e. going to more deeply bound states, the denominator decreases. This then results in a larger energy splitting between the pseudo-spin partners. For example for the orbit $\tilde{\ell}=3$ the energy splitting between the pseudo-spin partners $\left(1 g_{7 / 2}\right.$ and $\left.\left.2 d_{5 / 2}\right)\right)$ will be relatively larger as compared to that between $\left(2 g_{7 / 2}\right.$ and $\left.\left.3 d_{5 / 2}\right)\right)$. In addition, the bigger is the value of $\tilde{\ell}$ the larger is the splitting. For instance,the energy splitting between the pseudo-spin partners $\left(1 i_{11 / 2}\right.$ and $\left.2 g_{9 / 2}\right)$ corresponding to $\tilde{\ell}=5$ is relatively larger as compared to that between the partners $\left(2 g_{7 / 2}\right.$ and $\left.3 d_{5 / 2}\right)$ which corresponds to $\tilde{\ell}=3$, in the same major shell. Interestingly, the sign of the energy splittings between the partners of the neutron valence doublets is opposite to those of the neutron hole, proton particle and proton hole doublets.

The normalized single nucleon wave functions (both large $(f)$ and small $(g)$ components) 
are plotted for the pseudo-spin partners corresponding to the valence neutron pairs, the neutron hole pairs and valence proton pairs in Figs. 1 (b), (c) and (d) respectively. The phase of the lower components $(g)$ of one of the partners is reversed while plotting, in order to exemplify the differences in the lower components of the pseudo-spin partners. Clearly, the lower components are much smaller in magnitude as expected and are almost equal in magnitude. In the case of exact pseudo-spin symmetry, the lower component of the pseudospin partners should be identical (except for the phase). The very small differences between these $g$ 's which mainly appear around the surface are negligible for the pseudo-spin partners having very small binding energies.

Next we consider deformed systems and impose constraint on the quadrupole moment. Constrained relativistic Hartree calculations have been carried out for the nucleus ${ }^{154} \mathrm{Dy}$. The coupled differential equations for the spinors and the meson fields are given in Ref. [13]. They have been solved by expanding the spinors and the meson fields in terms of anisotropic oscillator wave functions. Numerical details are given in Refs. [13] and [18]. Pairing correlations are treated in the constant gap approximation and the Lagrangian parameter set NL3 [17] is used. The calculated potential energy surface is shown in Fig. 22. The value of the calculated ground state deformation parameter $\beta_{2}$ is 0.202 which is to be compared with 0.237 , the corresponding experimental value. The calculated ground state binding energy $1262.95 \mathrm{MeV}$ differs from the corresponding experimental value by merely $1.2 \mathrm{MeV}$.

The energies of the bound neutron pairs of orbitals corresponding to pseudo-spin doublets are plotted against the deformation $\beta_{2}$ ranging from 0.0 to 0.5 in Fig. 旬. The asymptotic Nilsson quantum numbers $\left[N, n_{3}, \Lambda, \Omega\right]$ are good for large values of the deformation $\beta_{2}$. The pseudo-spin doublets $\left[\tilde{N}, \tilde{n}_{3}, \tilde{\Lambda}, \tilde{\Omega}=\tilde{\Lambda} \pm 1 / 2\right]$ [5] are indicated by $\left[\tilde{N}, \tilde{n}_{3}, \tilde{\Lambda}\right] \uparrow$ and $\downarrow$ in the figure. For zero deformation $\left(\beta_{2}=0\right)$ the orbitals are indicated by the corresponding spherical states. The figure reveals the following:

(i) The energy splitting between the pseudo-spin partners is smaller for the valence orbitals 
and for the partners just below the Fermi surface.

(ii) This energy difference is relatively larger for the partners having larger pseudo-spin angular momentum $(\tilde{\ell})$.

(iii) In general, this separation stays almost constant and does not vary with deformation after reasonable value of $\beta_{2}$.

(iv) The energy difference between the $\downarrow$ and the $\uparrow$ partners always remains positive except for $[\mathbf{4 0} 4]$, where there is crossing at around $\beta=0.3$. Such a crossing is not very unusual, it has also been observed in Ref. [5].

These systematics are consistent with those observed in the spherical case above. A similar plot for the proton pseudo-spin doublets shown in Fig. 1 reveals identical systematics as those observed for the neutron case (Fig. 3). It is interesting to note that in Ref. [5] the energy difference between the valence neutron pseudo-spin partners is negative (opposite to ours) while it has the same sign as ours for protons. This may be due to the negative value obtained for $V_{\ell s}$, the strength of the pseudo-spin orbit interaction, from the Nilsson parameterization for $82<N<126$.

Similar calculations have also been carried out for other spherical and deformed nuclei and they show identical systematics. The conclusions presented here, are therefore rather general.

In conclusion, it is shown in the relativistic mean field framework that quasi-degenerate pseudo-spin doublets do exist near the fermi surface for both spherical and deformed nuclei. The pseudo-spin symmetry is restored better and better as one moves closer to the continuum limit. These conclusions confirm the findings of Ginocchio 4,10 .

One of the authors (G.A.L) acknowledges support from the DAAD. The work is also supported in part by the Bundesministerium für Bildung und Forschung under the project 06 TM 875. 
[1] K.T. Hecht, A. Adler; Nucl. Phys. A137, 129 (1969).

[2] A. Arima, M. Harvey, K. Shimizu; Phys. Lett. B30, 517 (1969).

[3] R.D. Ratna Raju, J.P. Draayer, K.T. Hecht; Nucl. Phys. A202, 433 (1973).

[4] J. N. Ginocchio, Phys. Rev. Lett. 78, 436 (1997).

[5] A. Bohr, I. Hamamoto and B. R. Mottelson, Phys. Scr. 26, 267 (1982).

[6] B. Mottelson, Nucl. Phys. A522, 1 (1991).

[7] C. Bahri, J. P. Draayer ans S. A. Moszkowski, Phys. Rev. Lett. 68, 2133 (1992).

[8] O. Castanos, M. Moshinsky, and C. Quesne, Phys. Letts. B277, 238 (1992).

[9] A. L. Blokhin, C. Bahri, J. P. Draayer, J. Phys. A29, 2039 (1996).

[10] J. N. Ginocchio and A. Leviatan, Los Alamos Preprint LA-UR-97-4357

[11] B. D. Serot and J. D. Walecka, Adv. Nucl. Phys. 16, 1 (1986), and references cited therein.

[12] P. G. Reinhard, Rep. Prog. Phys. 52, 439 (1989).

[13] Y. K. Gambhir, P. Ring and A. Thimet, Ann. Phys. (N.Y.) 198, 132 (1990), and references cited therein.

[14] P. Ring, Prog. Part. Nucl. Phys. 37, 193 (1996).

[15] J. N. Ginocchio and D. G. Madland, Los Alamos Preprint LA-UR-97-3921

[16] J. Meng, K. Sugawara-Tanabe, S. Yamaji, P. Ring, and A. Arima, preprint RIKEN 1997

[17] G. A. Lalazissis, J. König and P. Ring, Phys. Rev. C55, 540 (1997).

[18] P. Ring, Y.K. Gambhir, and G.A. Lalazissis Comp. Phys. Comm. 105, 77 (1997). 
FIG. 1. Pseudo-spin splitting in the spherical nucleus ${ }^{208} \mathrm{~Pb}$ : (a) single particle spectra in the vicinity of the Fermi surface for neutrons $\nu$ and protons $(\pi)$ and large $(f)$ and small $(g)$ components of the Dirac wave functions for the pseudo-spin doublets $\nu 2 \tilde{d}(\mathrm{~b}), \nu 2 \tilde{f}$ (c) and $\pi 2 \tilde{g}$ (d)

FIG. 2. Energy surface of the deformed nucleus ${ }^{154}$ Dy as function of the quadrupole moment $q$ in units of barn

FIG. 3. Single particle energies of the deformed Dirac equation for the neutrons in the nucleus ${ }^{154} \mathrm{Dy}$ as a function of the quadrupole deformation parameter $\beta_{2}$. Asymptotic pseudo-spin quantum numbers are given and the pseudo-spin partners are indicated by arrows $\uparrow$ and $\downarrow$

FIG. 4. Single particle energies for protons in ${ }^{154}$ Dy, for details see Fig. 3 

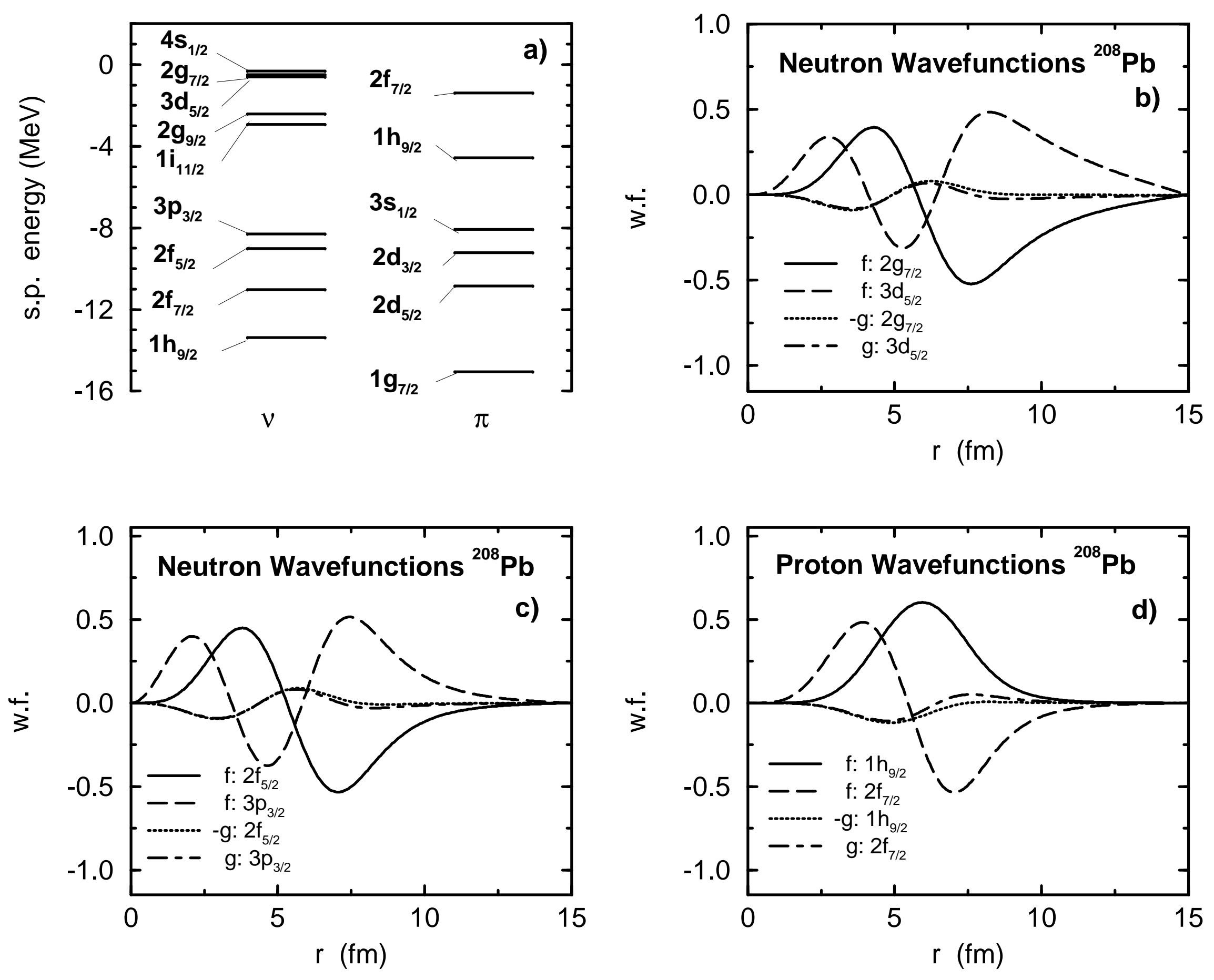


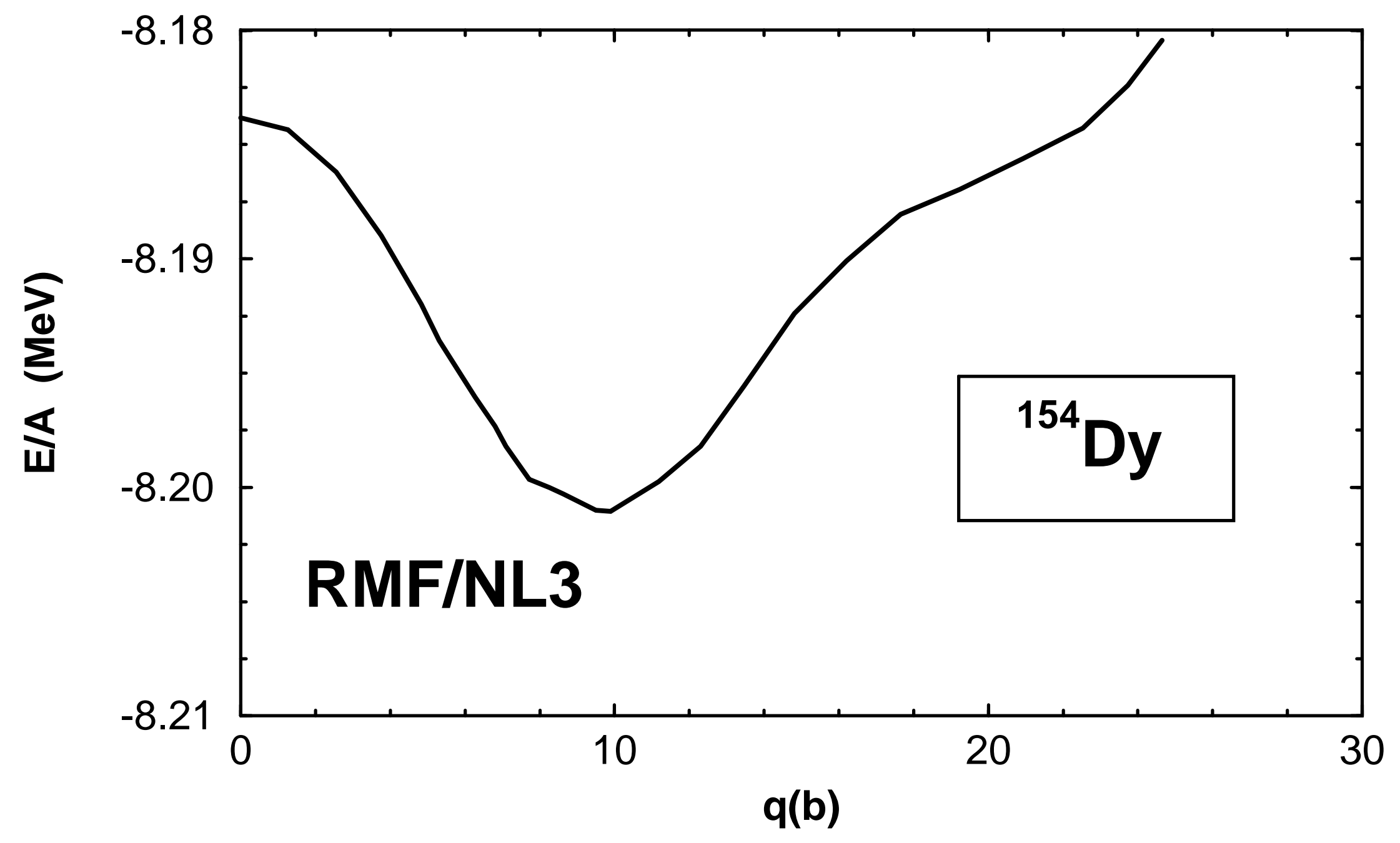




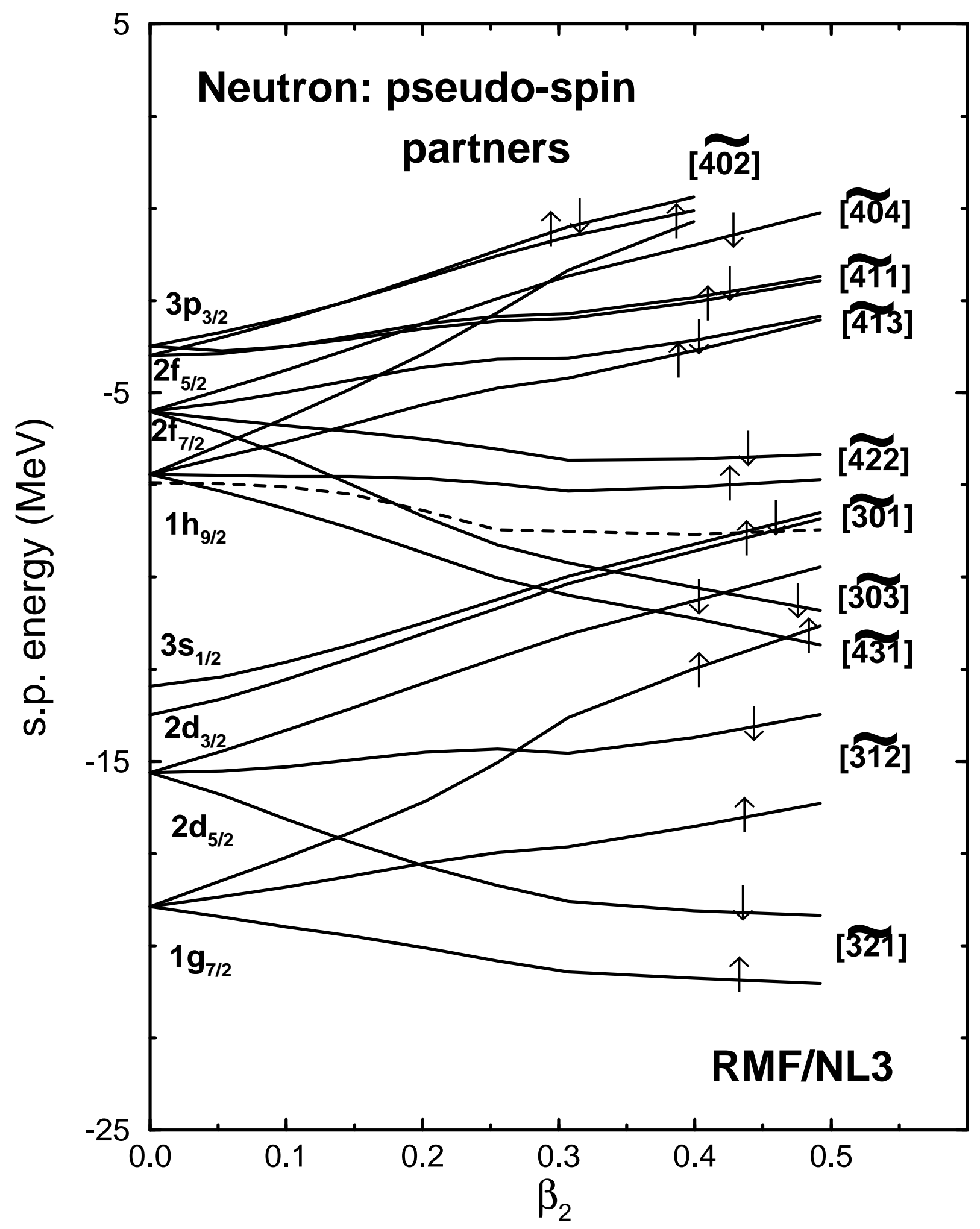




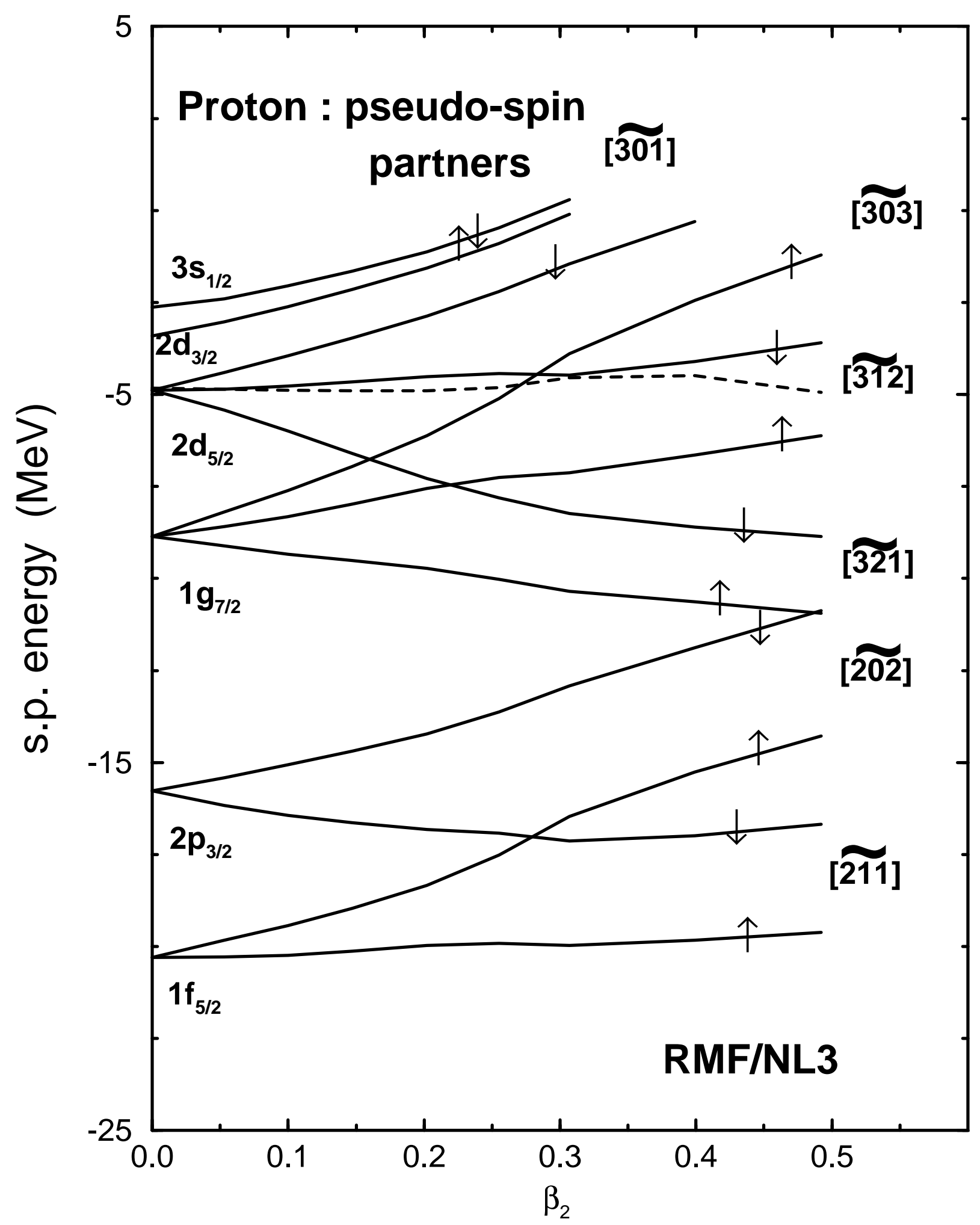

